\title{
A descrição do método LAMBDA utilizado na solução das ambiguidades
}

\author{
Crislaine Menezes da Silva, \\ Programa de Mestrado em Matemática Aplicada e Computacional - PósMac, FCT, UNESP \\ 19060-900, Presidente Prudente, SP \\ E-mail: crismenezes_@live.com
}

Daniele Barroca Marra Alves

Departamento de Cartografia, FCT, UNESP

19060-900, Presidente Prudente, SP

E-mail: danibarroca@fct.unesp.br

\author{
Eniuce Menezes de Souza \\ Departamento de Estatística, UEM \\ 87020-900, Maringa, PR \\ E-mail: emsouza@uem.br
}

Resumo: O posicionamento GNSS (Global Navigation Satellite System) tem apresentado resultados com alta precisão, oferecendo informações de posição cada vez mais acuradas. Atualmente, alguns métodos de posicionamento já apresentam erros de poucos centímetros, algumas vezes até milímetros. Eles utilizam a observável fase da onda portadora, que atinge uma acurácia, cerca de cem vezes melhor do que a outra observável, a pseudodistância. Mas, a acurácia centimétrica ocorre apenas quando as ambiguidades da fase da onda portadora são resolvidas corretamente como valores inteiros. As ambiguidades da fase da onda portadora são os números de ciclos inteiros entre as antenas do satélite e do receptor, na primeira época de dados. Elas são inseridas como parâmetro na equação de observação da fase da onda portadora. A solução correta das ambiguidades como valores inteiros é um desafio, além de ser uma peça fundamental nos métodos de posicionamento que utilizam a fase da onda portadora. Por esta razão tem sido fonte de pesquisa no posicionamento GNSS. No Brasil, pode-se citar a pesquisa desenvolvida por [2] para posicionamento relativo de linhas de bases curtas. O problema da solução das ambiguidades como valores inteiros pode ser dividido em duas partes: a estimação e a validação. A estimação consiste em calcular as ambiguidades em valores inteiros e a validação é utilizada para verificar se o valor encontrado pode ser utilizado ou não. Um método para estimação das ambiguidades inteiras, muito utilizado pela comunidade civil e científica internacional, é o método LAMBDA (Least-squares AMBiguity Decorrelation Adjustment). Neste método a estimativa inteira das ambiguidades por mínimos quadrados é calculada em duas etapas. Primeiramente é realizada uma decorrelação das ambiguidades, através da transformação Z. E, em segundo lugar, o problema de minimização é resolvido por uma procura discreta em uma região elipsoidal, através do espaço de procura das ambiguidades. Este artigo tem como objetivo descrever os modelos matemáticos empregados no método LAMBDA, o qual é utilizado no processo de solução das ambiguidades inteiras.

Palavras-chave: Método LAMBDA, Ambiguidades, GNSS, Geodésia

\section{Introdução}

A medida de fase da portadora é obtida a partir da diferença entre as fases do sinal gerado no receptor e a recebida do satélite no instante da medida. Assim, o receptor acumula o número de ciclos que chegam, resultando em uma medida contínua. Uma vez que esta medida somente pode ser obtida após a aquisição do sinal, o número inteiro de ciclos entre o satélite e o receptor 
permanece desconhecido. O modelo matemático para a fase da onda portadora é dado, segundo $[3]$,

$$
\phi_{r}^{s}=f\left(\frac{\rho_{r}^{s}-I_{r}^{s}+T_{r}^{s}+d m}{c}\right)+f\left(d t_{r}-d t^{s}\right)+\left(\phi^{s}\left(t_{0}\right)-\phi_{r}\left(t_{0}\right)\right)+N_{r}^{s}+\varepsilon_{r}^{s},
$$

onde $f$ é a frequência da fase; $\rho_{r}^{s}$ é distância geométrica entre o satélite e o receptor, calculada entre o instante de transmissão e recepção do sinal; $I_{r}^{s}$ é o erro causado pela ionosfera; $T_{r}^{s}$ é o erro causado pela troposfera; $d m$ são os efeitos de multicaminho; $c$ é a velocidade da luz no vácuo; $d t_{r}$ é o erro do relógio do receptor em relação ao tempo GPS; $d t^{s}$ é o erro do relógio do satélite em relação ao tempo GPS; $\phi_{r}\left(t_{0}\right)$ é a fase gerada no receptor na época de referência $t_{0} ; \phi^{s}\left(t_{0}\right)$ é a fase transmitida pelo satélite na época de referência $t_{0} ; N_{r}^{s}$ é a ambiguidade e $\varepsilon_{r}^{s}$ são outros erros não modelados. Os parâmetros desconhecidos em (1) são as ambiguidades e as coordenadas da estação de interesse. É possível notar na equação (1) que o sinal GPS sofre a influência de muitos erros, e uma estratégia para atenuá-los é tomar as duplas diferenças (DD) da observável. Elas são formadas a partir da combinação de observações simultâneas coletadas de dois receptores para dois satélites, envolvendo, desta forma, pelo menos dois receptores e pelo menos dois satélites. A equação da DD da fase da onda portadora é escrita como [3]:

$$
\Delta \phi_{1,2}^{1,2}=\frac{f}{c}\left(\Delta \rho_{1,2}^{1,2}\right)+N_{1,2}^{1,2}+v_{\Phi_{D D}},
$$

onde $N_{1,2}^{1,2}$ é a ambiguidade da DD, dada como $\left(N_{1}^{1}-N_{2}^{1}\right)-\left(N_{1}^{2}-N_{2}^{2}\right)$ e $v_{\Phi_{D D}}$ são os outros erros não modelados da DD da fase da onda portadora. Observe que em (2) não aparecem os erros referentes a ionosfera, troposfera e multicaminho que eram apresentados em (1), pois foram minimizados. Estes erros são eliminados se são utilizadas linhas de base curtas. ${ }^{1}$

\section{O método LAMBDA}

Foi um método proposto por [5] que visa estimar as ambiguidades inteiras na medida de fase da onda portadora. Para isso, utiliza-se um modelo matemático simples para linhas de base curtas. As equações de observação de dupla diferença linearizadas são dadas pelo seguinte sistema linear de equações, segundo [1]:

$$
y=A a+B b+\epsilon,
$$

onde $y$ é o vetor de dupla diferenças observadas menos as calculadas, $a$ é o vetor de ambiguidades inteiras da $\mathrm{DD}, b$ é o vetor que contem os parâmetros referentes a linha de base, A e B são matrizes design para as ambiguidades e linha de base,respectivamente e $\epsilon$ é o vetor de erros não modelados. A matriz de variância-covariância (MVC) da observável y é $Q_{y}$, e é simétrica e definida positiva. A partir da equação (3), o método LAMBDA, utiliza o método dos mínimos quadrados para calcular $a$ e $b$. O critério de minimização que visa resolver (3) é, de acordo com [6]:

$$
\min _{a, b}\|y-B b-A a\|_{Q_{y}}^{2},
$$

onde $\|\cdot\|_{Q_{y}}^{2}=(.)^{T} Q_{y}^{-1}($.$) com b \in R^{m}$ e $a \in Z^{m}$. A estimação dos parâmetros é feito em três etapas. São elas: a solução float, a estimação das ambiguidades inteiras e a solução fixa. Embora todas as etapas sejam importantes, este trabalho é concentrado na etapa da estimação das ambiguidades inteiras. A primeira etapa, a da solução float, consiste em minimizar (4) com $a \in R^{m}, b \in R^{m}$ utilizando o método dos mínimos quadrados comum. Obtendo como resultado uma estimativa $\hat{a}$ e $\hat{b}$ reais. A segunda etapa, a estimação das ambiguidades inteiras, consiste em resolver o problema de minimização, segundo [6]:

$$
\min _{a}\|\hat{a}-a\|_{Q_{\hat{a}}}^{2},
$$

$\operatorname{com} a \in Z^{m}$.

\footnotetext{
${ }^{1}$ Segundo [4], linha de base é a distância entre dois receptores GPS. São consideradas linhas de base curtas, linhas de base cujo comprimento não ultrapassa $20 \mathrm{~km}$, dependendo das condições ionosféricas.
} 


\subsection{A estimação das ambiguidades inteiras}

Esta etapa consiste na minimização de (5), resultando em uma estimativa inteira do vetor de ambiguidades $\stackrel{\vee}{a}$. Podemos reescrever (5), como sendo:

$$
\min _{a}(\hat{a}-a)^{T} Q_{\hat{a}}^{-1}(\hat{a}-a) .
$$

Na prática, a procura pela solução de (6) não é realizada em todo o conjunto dos números inteiros $Z^{m}$, em vez disso ela consiste em uma busca discreta dentro de um hiper-elipsóide n-dimensional. Esta região é tomada como base e a busca é então realizada, segundo [6]:

$$
(\hat{a}-a)^{T} Q_{\hat{a}}^{-1}(\hat{a}-a) \leq \chi^{2} .
$$

Este hiper-elipsóide é denominado como espaço de procura das ambiguidades. Ele é centrado na estimativa float das ambiguidades $\hat{a}$ e sua orientação e achatamento estão em função da MVC $Q_{\hat{a}}$. Além disso, o seu volume pode ser controlado ajustando o valor de $\chi^{2}$.

\subsection{Estimativa sequencial condicional por mínimos quadrados}

Na seção anterior não mostramos como resolver (6). O método mais trivial da estimação dos inteiros seria a aproximação para o inteiro mais próximo. Infelizmente, no geral, este tipo solução não dá a respota correta para (6). Isso ocorre apenas quando a MVC $Q_{\hat{a}}$ é diagonal, ou seja, quando as ambiguidades são totalmente decorrelacionadas. Assim, se $Q_{\hat{a}}$ é diagonal, obtemos uma mudança em (6), tornando-se, de acordo com [6]:

$$
\underset{a_{1}, \ldots, a_{m}}{\operatorname{minimizar}} \sum_{i=1}^{m} \frac{\left(\hat{a}_{i}-a_{i}\right)}{\sigma_{\hat{a}_{i}}^{2}} .
$$

Mas, na prática as ambiguidades são muito correlacionadas e a MVC não é diagonal. Deste forma, devemos diagonalizar a MVC preservando o que acontece em (8), ou seja, que as ambiguidades individuais possam ser atribuídas aos quadrados individuais. Para isso, utilizaremos o ajuste sequencial condicional por mínimos quadrados $\hat{a}_{i \mid I}, i=1, \ldots, m$. A estimativa condicional $\hat{a}_{i \mid I}$ é a estimativa para $a_{i}$ condicionada a $a_{i-1}$. Esta estimativa para $i$ é dada como, [6]:

$$
\hat{a}_{i \mid I}=\hat{a}_{i}-\sum_{j=1}^{i-1} \frac{\sigma_{\hat{a}_{i} \hat{a}_{j \mid J}}}{\sigma_{\hat{a}_{j \mid J}}^{2}}\left(\hat{a}_{j \mid J}-a_{j}\right),
$$

onde $\sigma_{\hat{a}_{i} \hat{a}_{j \mid J}}$ é a covariância entre $\hat{a}_{i}$ e $\hat{a}_{j \mid J}$. Agora, como o ajuste sequencial condicional das ambiguidades são decorrelacionados, podemos substituir (9) em (6), e assim obter, segundo [6]:

$$
\underset{a_{1}, \ldots, a_{m}}{\operatorname{minimizar}} \sum_{i=1}^{m} \frac{\left(\hat{a}_{i \mid I}-a_{i}\right)^{2}}{\sigma_{\hat{a}_{i \mid I}}^{2}} .
$$

Utilizando (10), podemos agora reescrever (7) como sendo:

$$
\sum_{i=1}^{m} \frac{\left(\hat{a}_{i \mid I}-a_{i}\right)^{2}}{\sigma_{\hat{a}_{i \mid I}}^{2}} \leq \chi^{2} .
$$

\subsection{A Transformação Z}

O método LAMBDA visa estimar as ambiguidades inteiras através de uma decorrelação nas ambiguidades, que é realizada através de uma reparametrização. A reparametrização é feita da seguinte forma, segundo [1]:

$$
\begin{aligned}
& z=Z^{T} a \\
& \hat{z}=Z^{T} \hat{a} \\
& Q_{\hat{z}}=Z^{T} Q_{\hat{a}} Z \\
& \stackrel{\vee}{a}=Z^{-T} \stackrel{\vee}{z},
\end{aligned}
$$


onde Z é uma transformação das ambiguidades admissível. Com isso, podemos reescrever (6), de uma forma equivalente, obtendo:

$$
\min _{z}(\hat{z}-z)^{T} Q_{z}^{-1}(\hat{z}-z),
$$

em que $z \in Z^{m}$. Da mesma forma, o espaço de procura das ambiguidades (7), é transformado em, conforme [6]:

$$
(\hat{z}-z)^{T} Q_{\hat{z}}^{-1}(\hat{z}-z) \leq \chi^{2} .
$$

A fim de responder a questão sobre como construir a transformação das ambiguidades admissível $Z^{T}$, construiremos agora a transformação para o caso bidimensional. Para isso, sejam as ambiguidades e sua MVC dadas como:

$$
\begin{aligned}
& a=\left(\begin{array}{c}
\hat{a}_{1} \\
\hat{a}_{2}
\end{array}\right) \\
& Q_{\hat{a}}=\left(\begin{array}{cc}
\sigma_{\hat{a}_{1}}^{2} & \sigma_{\hat{a}_{1} \hat{a}_{2}} \\
\sigma_{\hat{a}_{2} \hat{a}_{1}} & \sigma_{\hat{a}_{2}}^{2}
\end{array}\right) .
\end{aligned}
$$

Quando (9) é escrita na forma matricial, obtemos, como descrito por [6]:

$$
\left(\begin{array}{c}
\hat{a}_{1} \\
\hat{a}_{2 \mid 1}
\end{array}\right)=\left(\begin{array}{cc}
1 & 0 \\
\frac{-\sigma_{\hat{a}_{2} \hat{a}_{1}}}{\sigma_{\hat{a}_{1}}^{2}} & 1
\end{array}\right)\left(\begin{array}{c}
\hat{a}_{1} \\
\hat{a}_{2}
\end{array}\right) .
$$

Note que (16) distingue de (9) apenas por $a_{1}$, que por razões de conveniência será tratado como nulo. Se $\frac{-\sigma_{\hat{a}_{2} \hat{a}_{1}}}{\sigma_{\hat{a}_{1}}}$ for inteiro a matriz acima seria uma transformação das ambiguidades admissível, mas em geral, ele não o é. Contudo, isto pode ser resolvido facilmente aproximando $\frac{-\sigma_{\hat{a}_{2} \hat{a}_{1}}}{\sigma_{\hat{a}_{1}}^{2}}$ por $-\left[\frac{-\sigma_{\hat{a}_{2} \hat{a}_{1}}}{\sigma_{\hat{a}_{1}}}\right]$ onde [.] é o "arredondamento para o inteiro mais próximo". Obtendo:

$$
\left(\begin{array}{c}
\hat{a}_{1} \\
\hat{a}_{2^{\prime} \mid 1}
\end{array}\right)=\left(\begin{array}{cc}
1 & 0 \\
-\left[\frac{\sigma_{\hat{a}_{2} \hat{a}_{1}}}{\sigma_{\hat{a}_{1}}^{2}}\right] & 1
\end{array}\right)\left(\begin{array}{c}
\hat{a}_{1} \\
\hat{a}_{2}
\end{array}\right),
$$

onde $\hat{a}_{2^{\prime} \mid 1}$ é uma aproximação para $\hat{a}_{2 \mid 1}$. Observe que em (16) optamos por deixar $\hat{a}_{1}$ inalterado. Mas se ao invés de $\hat{a}_{1}$ preferirmos manter $\hat{a}_{2}$ obteríamos no lugar de (9), a seguinte forma matricial, conforme [6]:

$$
\left(\begin{array}{c}
\hat{a}_{1 \mid 2} \\
\hat{a}_{2}
\end{array}\right)=\left(\begin{array}{cc}
1 & -\frac{\sigma_{\hat{a}_{1} \hat{a}_{2}}}{\sigma_{\hat{a}_{2}}} \\
0 & 1
\end{array}\right)\left(\begin{array}{c}
\hat{a}_{1} \\
\hat{a}_{2}
\end{array}\right) .
$$

Para tornar (18) admissível, faremos agora o que fizemos com (17), arredondaremos $-\frac{\sigma_{\hat{a}_{1} \hat{a}_{2}}}{\sigma_{\hat{a}_{2}}^{2}}$ para o seu inteiro mais próximo, obtendo assim, [6]:

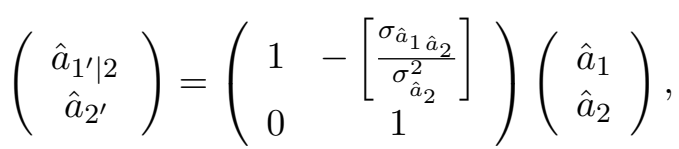

onde $\hat{a}_{1^{\prime} \mid 2}$ é uma aproximação para $\hat{a}_{1 \mid 2}$ e $\hat{a}_{2^{\prime}}$ é uma aproximação para $\hat{a}_{2}$. Com estes passos, contruímos duas transformações admissíveis. Portanto, podemos agora montar nossa transformação das ambiguidades, utilizando (17) e (19) dada como, [6]:

$$
Z^{T}=Z_{2}^{T} Z_{1}^{T}=\left(\begin{array}{cc}
1 & 0 \\
-\left[\frac{\sigma_{\hat{a}_{2} \hat{a}_{1}}}{\sigma_{\hat{a}_{1}}^{2}}\right] & 1
\end{array}\right)\left(\begin{array}{cc}
1 & -\left[\frac{\sigma_{\hat{a}_{1} \hat{a}_{2}}}{\sigma_{\hat{a}_{2}}^{2}}\right] \\
0 & 1
\end{array}\right)
$$

Com os procedimentos acima, é obtida a solução para o problema (13), e após isso, encontra-se $\stackrel{\vee}{a}$. 


\subsection{Simulação}

Uma simulação de decorrelação 2D será apresentada, utilizando um exemplo sintético. Para isso, consideram-se o vetor $a$ das ambiguidades e os coeficientes da sua MVC $Q_{\hat{a}}$, conforme (15):

$$
\hat{a}=\left(\begin{array}{c}
5.54 \\
3.1
\end{array}\right) Q_{\hat{a}}=\left(\begin{array}{cc}
6.29 & 5.978 \\
5.978 & 6.2920
\end{array}\right)
$$

Utilizando (19), obtemos $Z_{1}^{T}$ e $Q_{Z_{1}^{T}}$ :

$$
Z_{1}^{T}=\left(\begin{array}{cc}
1 & -1 \\
0 & 1
\end{array}\right) Q_{Z_{1}^{T}}=\left(\begin{array}{cc}
0.6260 & -0.3140 \\
-0.3140 & 6.2920
\end{array}\right)
$$

Agora utiliza-se (17)para obter $Z_{2}^{T}$ :

$$
Z_{2}^{T}=\left(\begin{array}{ll}
1 & 0 \\
1 & 1
\end{array}\right) .
$$

Com (21) e (22), encontra-se agora $Z^{T}$ :

$$
Z^{T}=\left(\begin{array}{cc}
1 & -1 \\
1 & 0
\end{array}\right)
$$

Aplicando (12), juntamente com (2), obtemos:

$$
\begin{gathered}
\hat{z}=Z^{T} a=\left(\begin{array}{cc}
1 & -1 \\
1 & 0
\end{array}\right)\left(\begin{array}{c}
5.54 \\
3.1
\end{array}\right) \\
\hat{z}=\left(\begin{array}{c}
2.44 \\
5.54
\end{array}\right) \\
\stackrel{v}{z}=\left(\begin{array}{c}
2.44 \\
{[5.54}
\end{array}\right)=\left(\begin{array}{c}
2 \\
6
\end{array}\right) \\
Q_{\hat{z}}=Z^{T} Q_{\hat{a}} Z=\left(\begin{array}{cc}
1 & 0 \\
1 & 1
\end{array}\right)\left(\begin{array}{cc}
6.29 & 5.978 \\
5.978 & 6.2920
\end{array}\right)\left(\begin{array}{ll}
1 & 1 \\
0 & 1
\end{array}\right) \\
Q_{\hat{z}}=\left(\begin{array}{cc}
0.6260 & 0.3120 \\
0.3120 & 6.29
\end{array}\right) .
\end{gathered}
$$

Note que $Q_{\hat{z}}$ em (24) é menos correlacionada do que $Q_{\hat{a}}$ inicial. Por fim, tem-se:

$$
\begin{aligned}
& \stackrel{\vee}{a}=Z^{-T} \stackrel{\vee}{z}=\left(\begin{array}{cc}
0 & 1 \\
-1 & 1
\end{array}\right)\left(\begin{array}{l}
2 \\
6
\end{array}\right) \\
& \stackrel{\vee}{a}=\left(\begin{array}{l}
6 \\
4
\end{array}\right) .
\end{aligned}
$$

\section{Conclusão}

No presente trabalho, mostrou-se o conceito de solução das ambiguidades, bem como o método LAMBDA. Este método realiza a estimação das ambiguidades inteira da dupla diferença. Para isso, ele aplica a transformação Z nas ambiguidades, cuja matriz de transformação é inteiro e sua inversa também o é, a fim de decorrelacioná-las antes de iniciar a busca. O problema então é abordado através de uma busca discreta sobre uma região elipsoidal, que permite encontrar a solução inteira procurado. O resultado é baseado nas ambiguidades float e sua MVC, e fornece uma estimativa inteira por mínimos quadrados para as ambiguidades. 


\section{Agradecimentos}

Este trabalho está sendo desenvolvido com recursos financeiros da FAPESP (Fundação de Amparo à Pesquisa do Estado de São Paulo) mediante atribuição de bolsa de Mestrado à primeira autora. Processo no 2013/06325-9.

\section{Referências}

[1] P. DE JONGE; C. C. J. M. TIBERIUS, "The LAMBDA method for integer ambiguity estimation: implementation aspects", T.U.Delft - internal report, Delft, 1996

[2] W.C. MACHADO,"Solução rápida das ambiguidades GPS para aplicações no posicionamento relativo de linhas de base curtas". 2001. 128f. Dissertação(Mestrado em Ciências Cartográficas)-Faculdade de Ciências e Tecnologia, Universidade Estadual Paulista, Presidente Prudente.

[3] J. F. G. MONICO,"Posicionamento pelo GNSS: Descrição, fundamentos e aplicações". 2. ed. São Paulo: Editora UNESP, 2008. 476 p.

[4] G. SEEBER, "Satellite Geodesy : Foundations, Methods and Aplications". 2 ed.,Berlin, New-York: Walter de Gruyter, 2003.

[5] P. J. G. TEUNISSEN, Least-squares estimation of the integer GPS ambiguities. In:IAG GENERAL MEETING, 1993, Beijing, China. "Proceedings... Beijing": IAG,1993.

[6] P. J. G. TEUNISSEN, GPS Carrier Phase Ambiguity Fixing Concepts. In: TEUNISSEN, P. J. G.; KLEUSBERG,A."GPS for Geodesy". 2ed. Berlin: Springer Verlage, 1998, p.271-318. 